\title{
INTERNET Y AGRESIVIDAD EN ESTUDIANTES DE SECUNDARIA DEL PERÚ
}

\section{INTERNET AND AGGRESIVITY IN HIGH SCHOOL STUDENTS OF PERU}

\author{
Yarlequé Chocas Luis Alberto; Javier Alva Leda; Nuñez Llacuachaqui Edith Rocío; \\ Navarro García Linda Loren; Matalinares Calvet María Luisa
}

\section{RESUMEN}

Se aborda la influencia que tiene la adicción a Internet sobre la agresividad que presentan los estudiantes de educación secundaria del Perú. Se utilizó el método descriptivo, con diseño causal comparativo se evaluó a 2, 225 estudiantes de secundaria, de 13 departamentos de la costa, sierra y selva, en su agresividad y la adicción a internet. Los resultados mostraron, entre otras cosas que, la mayoría de los estudiantes que presentan altos niveles de adicción a la internet tienden a ser más agresivos que los que presentan bajos niveles, lo cual conduce a suponer que la adicción a la internet es uno de los multiples factores que acicatea la agresividad que muestran los jóvenes.

Palabras clave: adicción a la internet, agresividad.

\begin{abstract}
It is about the influence of Internet addiction on the aggressiveness of students of secondary education of Peru. We used descriptive method with design comparative -causal, 2, 225 high school students of 13 departments from the coast, highlands and jungle were evaluated in their aggressiveness and Internet addiction. The results showed, among other things, that most students with high levels of internet addiction tend to be more aggressive than those with low levels, which leads to assume that internet addiction is one of the multiple factors that spurs aggression of students.
\end{abstract}

Keywords: internet addiction, aggression.

\section{INTRODUCCIÓN}

La internet es una herramienta que ha surgido en las últimas décadas. En el Perú su uso se ha difundido desde hace casi 20 años. Si es correctamente utilizada ofrece innumerables ventajas. Echeburúa y cols. (1998); Griffiths (1997); Young (1996) explican las consecuencias negativas relacionadas con el uso de Internet y proponen la existencia de un desorden de adicción a Internet similar a los problemas que aparecen con otras conductas adictivas (juego, sexo, trabajo, las compras, etc.).

Con el desarrollo de Internet y su gran crecimiento han aparecido también los primeros casos de psicopatología. El trastorno de dependencia de la red se ha conocido con muchos nombres: desorden de adicción a Internet (Goldberg, 1995 citado por Balaguer 2001), uso compulsivo de Internet (Morahan-Martin y Schumacker, 1997), o uso patológico de Internet (Young y Rodgers, en López, 2000). Echeburúa (1999) explica que el medio en el que se desenvuelve la adicción acarrea una serie de cambios psicológicos negativos como alteraciones del humor, ansiedad o impaciencia por la lentitud de las conexiones o por no encontrar lo que se busca o a quien se busca, estado de conciencia alterado, irritabilidad en caso de interrupción, incapacidad para salir del monitor, etc. El autor insiste en que el adicto se aísla del entorno y no presta atención a otros aspectos de las obligaciones sociales. A decir de Young (1999) los efectos negativos de la adicción se expresan en los ámbitos familiar, académico y profesional. También la BBC del Mundo (2011) informó que en Corea del Sur el número de adictos al internet ha aumentado y el parlamento aprobó una ley para restringir su uso entre menores de 18 años. En la Dirección de adicciones del Instituto Nacional de Salud Mental Honorio DelgadoHideyo Noguchi se viene atendiendo cada vez más personas con esta patología desde hace más de 10 años (Cruzado, Matos y Kendall, 2006). 
En lo que se refiere a la agresividad, Berkowitz (1996) la define como un "estado emocional que consiste en sentimientos de odio y deseos de dañar a otra persona, animal u objeto". Se manifiesta mediante ataques físicos o verbales hacia otro que generalmente es menor que el agresor; es decir, que una persona en mejores condiciones que otra trata de imponer sus reglas para lograr su propósito (Hurlock, 2000). Para Buss (1992), las respuestas agresivas poseen dos características: la descarga de estímulos nocivos y un contexto interpersonal; definiendo a la agresión como una reacción que descarga estímulos nocivos sobre otros organismos.

Por otro lado, es importante recordar que, el Perú era para el año 2005 el segundo en Latinoamérica en cuanto a tasa de penetración de internet $(11,5$ usuarios/100 habitantes) y el $66 \%$ de adolescentes de Lima accedían a internet regularmente, la mayoría a través de cabinas públicas (Cruzado, Matos y Kendall, 2006). Se puede inferir que este problema es cada vez más creciente en la población.

A este respecto, EscucharLeer fonéticamenteYoung y Rodgers (1998) realizaron un estudio para evaluar la relación entre la depresión y la adicción a Internet. Arai, González, Morales, Gutiérrez y Tettley (2008) en México, investigaron las consecuencias del uso de Internet en alumnos de preparatoria. En Asia, Yen, Ko, Yen, Chen, Chung y Chen (2008) investigaron los síntomas psiquiátricos, la adicción a Internet y el uso de sustancias. Herrera, Pacheco, Palomar y Zavala (2010) en México estudiaron la adicción al facebook y la baja autoestima, depresión y falta de habilidades sociales. Chain- Pinzon (2011) en Colombia, trabajó acerca de la relación entre la frecuencia de actividad física con las variables agresividad, impulsividad, internet y videojuegos en adolescentes colombianos. Lam, Contreras, Mori, Nizama y otros (2011) validaron un instrumento para evaluar la adicción al internet en adolescentes. En torno a la agresividad, Castrillon y Vieco (2002) en la Medellín, han evaluado las actitudes mediante las cuales los estudiantes universitarios justifican sus comportamientos agresivos y violentos. En España, Vacas (2002) investigó la relación entre la agresividad escolar y las dificultades de aprendizaje en una muestra de alumnos de primaria hasta el bachillerato. Sanz y González (2006) se han ocupado de la expresión de la ira en adolescentes Holguineros. Murcia, Reyes, Gómez, Medina, Paz y Fonseca (2007) analizaron los niveles de hostilidad y agresividad en adolescentes. Samper, Tur, Mestre y Cortés (2008) abordaron la agresividad y el afrontamiento en la adolescencia desde una perspectiva intercultural. En el Perú, Hernández (1988) realizó un estudio sobre la relación entre las actitudes parentales y la conducta agresiva de los preescolares. Velásquez, Arenas, Campos, Dioses, Gutiérrez, García, Elizalde y Montero investigaron la ansiedad y cólera en estudiantes de Lima y Trujillo con participación y no participación en actos Violentos. Rodríguez y Tincopa (2006) analizaron la relación entre el soporte social y agresividad en adolescentes infractores del centro juvenil "Trujillo". Oliveros, Figueroa, Mayorga, Cano y Quispe (2008) estudiaron la violencia escolar (bullyng) en colegios Estatales de Primaria del Perú. Cano, Gutiérrez y Nizama (2009) investigaron la tendencia a la violencia e ideación suicida en adolescentes escolares en una ciudad de la amazonía peruana. Amemiya, Oliveros, y Barrientos (2009) investigaron los factores de riesgo de violencia escolar (bullying) severa en colegios privados de tres zonas de la sierra del Perú. Matalinares, Arenas, Sotelo y col (2010) han estudiado la relación entre el clima familiar y la agresividad en estudiantes de secundaria. Más no se ha estudiado las relaciones causales entre la adicción a la red y la agresividad, es por ello que se formuló el siguiente problema ¿Influye la adicción a la internet en la agresividad en estudiantes de secundaria del Perú?

\section{MATERIAL Y MÉTODOS}

La presente investigación es de tipo aplicada y de nivel explicativo (Sánchez y Reyes, 2006). El método utilizado fue el descriptivo (Alarcón. 1991) con diseño causal comparativo (Sánchez y Reyes, 2006).

La población estuvo constituida por estudiantes de tercero a quinto de secundaria del Perú. La muestra por 2,225 estudiantes de tercero (750), cuarto (742) y quinto(733) de secundaria, de Instituciones Educativas Estatales, de ambos sexos, cuyas edades fluctuaban entre los 13 y 18 años procedentes de diferentes las regiones: Tacna, Ayacucho, Amazonas, Pucallpa, 
Huancavelica, Chiclayo Huancayo, Huánuco, Lima, San Martin, Trujillo, Piura y Cuzco.

Las variables de investigación fueron adicción a internet y agresividad, las de control: nivel educativo, género y regiones naturales.Las técnicas empleadas fueron la observación indirecta y la técnica psicométrica.

Los instrumentos utilizados fueron:1) Test de adicción al Internet propuesto por Young (1996) adaptado por Quispe (2011). El test es administración Individual 0 Colectiva. Está dirigido a adolescentes y jóvenes entre 13 y 18 años; consta de 20 reactivos agrupados en tres factores, mide el uso excesivo del internet. Este test está basado en los criterios diagnósticos relacionados con el juego patológico, la dependencia. Consta de 3 dimensiones: Saliencia, Descuido y Exceso. Ornella, Matalinares Y Deyvi (2012) encontraron que el test tiene una confiabilidad total moderada de 0.870 , así como se extrajo la confiabilidad por Costa con un 0.876 , sierra 0.878 y selva con 0.836 . Obteniendo 4 factores: tolerancia, uso excesivo, retirada y consecuencias negativas, con una varianza de $49.57 \%$.

Para medir la variable agresividad se uso el Cuestionario de Agresión (Aggression Questionnaire, $A Q$ ), propuesto por Buss y Perry (1992) y adaptado por Rodríguez (2002) para la población española. El test se administra de forma Individual y Colectiva. Está compuesta por 29 ítems que hacen relación a conductas y sentimientos agresivos. Estos ítems están codificados en una escala tipo Likert de cinco puntos. El cuestionario se estructura en cuatro sub-escalas denominadas: agresividad física, compuesta por nueve ítems, agresividad verbal, compuesta por cinco ítems, ira, compuesta por siete ítems y, finalmente, hostilidad, compuesta por ocho ítems. La Validez, se realizó a través de la técnica del análisis factorial exploratorio, se determinaron las cuatro sub-escalas dicha estructura factorial en una segunda muestra de sujetos a través del análisis factorial confirmatorio; 10 que añadió mayor validez de constructo a la estructura tetradimensional determinada en la primera muestra de estudio. La Confiabilidad se trabajó mediante los coeficientes de consistencia interna encontrados, éstos fueron satisfactorios: Alfa Cronbach $=0,86$ para la escala de la agresión física, Alfa Cronbach $=0,68$ para la escala de agresión verbal, Alfa Cronbach $=0,77$ para la escala de Ira, Alfa Cronbach=0,72 para la escala de Hostilidad y Alfa Cronbach $=0,88$ para la escala total. Se realizará la adaptación y baremación del test de Agresividad $A Q$ para el Perú.

\section{RESULTADOS}

Tabla 1. Resultados de la muestra por grado a nivel nacional en la escala de agresión

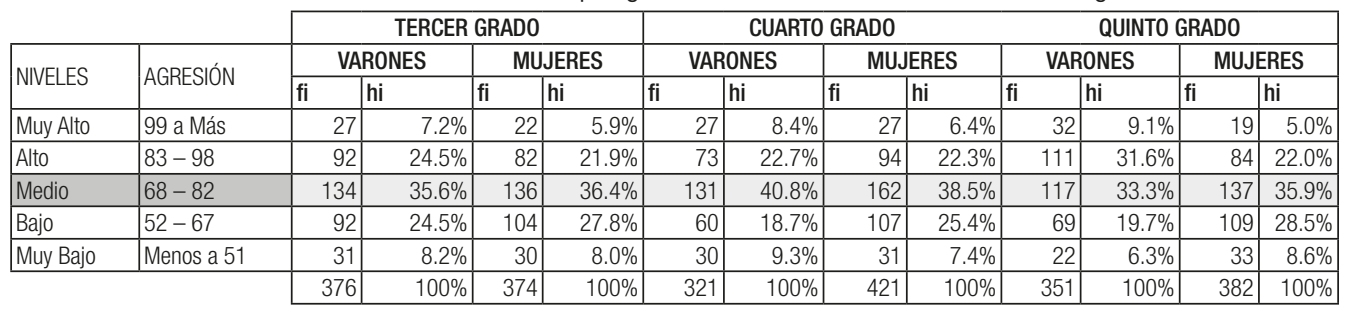

Tabla 2. Resultados de la muestra a nivel nacional en la escala de adicción a la internet

\begin{tabular}{|c|c|c|c|c|c|c|c|c|c|c|c|c|c|c|}
\hline & & \multirow{2}{*}{\multicolumn{6}{|c|}{ VARONES }} & & \multirow{2}{*}{\multicolumn{6}{|c|}{ MUJERES }} \\
\hline & & & & & & & & & & & & & & \\
\hline \multirow[t]{2}{*}{ Niveles } & \multirow[t]{2}{*}{ Intervalos } & \multicolumn{2}{|c|}{ TERCER GRADO } & \multicolumn{2}{|c|}{$\begin{array}{l}\text { CUARTO } \\
\text { GRAD0 }\end{array}$} & \multicolumn{2}{|c|}{ QUINTO GRADO } & \multirow[t]{2}{*}{ Intervalos } & \multicolumn{2}{|c|}{ TERCER GRADO } & \multicolumn{2}{|c|}{$\begin{array}{c}\text { CUARTO } \\
\text { GRAD0 }\end{array}$} & \multicolumn{2}{|c|}{ QUINTO GRADO } \\
\hline & & $\mathrm{fi}$ & hi & $\mathrm{fi}$ & hi & $\mathrm{fi}$ & hi & & $\mathrm{fi}$ & hi & $\mathrm{fi}$ & hi & $\mathrm{fi}$ & hi \\
\hline \begin{tabular}{|l|} 
Muy Alto \\
\end{tabular} & $51-95$ & 36 & $9.6 \%$ & 29 & $9.0 \%$ & 41 & $11.7 \%$ & $49-95$ & 33 & $9 \%$ & 34 & $8 \%$ & 36 & $9 \%$ \\
\hline \begin{tabular}{|l|} 
Alto \\
\end{tabular} & $50-40$ & 53 & $14.1 \%$ & 42 & $13.1 \%$ & 50 & $14.2 \%$ & $48-34$ & 41 & $11 \%$ & 47 & $11 \%$ & 56 & $15 \%$ \\
\hline \begin{tabular}{|l|} 
Medio \\
\end{tabular} & $39-16$ & 169 & $44.9 \%$ & 160 & $49.8 \%$ & 175 & $49.9 \%$ & $33-12$ & 179 & $48 \%$ & 193 & $46 \%$ & 167 & $44 \%$ \\
\hline Bajo & $15-8$ & 76 & $20.2 \%$ & 58 & $18.1 \%$ & 57 & $16.2 \%$ & 11 a 6 & 51 & $14 \%$ & 78 & $19 \%$ & 71 & $19 \%$ \\
\hline \multirow[t]{2}{*}{ Muy Bajo } & $7-0$ & 42 & $11.2 \%$ & 32 & $10.0 \%$ & 28 & $8.0 \%$ & $5-0$ & 70 & $19 \%$ & 69 & $16 \%$ & 52 & $14 \%$ \\
\hline & & 376 & $100 \%$ & 321 & $100 \%$ & 351 & $100 \%$ & & 374 & $100 \%$ & 421 & $100 \%$ & 382 & $100 \%$ \\
\hline
\end{tabular}




\section{HIPÓTESIS}

$\mathrm{H}_{0} \mathrm{No}$ existen diferencias significativas entre los puntajes en la prueba de agresividad de los varones con altos y bajos niveles de adicción a la internet.

$\mathrm{H}_{1}$ Existen diferencias significativas entre los puntajes en la prueba de agresividad de los varones con altos y bajos niveles de adicción a la internet.

Tabla 3: Comparación de medias en la prueba de agresividad de los varones con altos y bajos niveles de adicción a la internet

\begin{tabular}{|c|c|c|c|c|c|c|c|c|}
\hline X1 & X2 & S1 & S2 & Gl & Nc & Zt & Zp & Diagnóstico \\
\hline 50,61 & 8,35 & 9,77 & 3,86 & 522 & 0,05 & 1,96 & 2,86 & Significativo \\
\hline
\end{tabular}

$\mathrm{H}_{0}$ No existen diferencias significativas entre los puntajes en la prueba de agresividad de las mujeres con altos y bajos niveles de adicción a la internet.

$\mathrm{H}_{2}$ Existen diferencias significativas entre los puntajes en la prueba de agresividad de las mujeres con altos y bajos niveles de adicción a la internet.

Tabla 4: Comparación de medias en la prueba de agresividad de las mujeres con altos y bajos niveles de adicción a la internet

\begin{tabular}{|c|c|c|c|c|c|c|c|c|}
\hline X1 & X2 & S1 & S2 & Gl & Nc & Zt & Zp & Diagnóstico \\
\hline 46,16 & 4,37 & 12,72 & 2,59 & 586 & 0,05 & 1,96 & 9,34 & Significativo \\
\hline
\end{tabular}

\section{DISCUSIÓN}

Los resultados expuestos, muestran un panorama interesante: cuando la muestra se analiza por grados de instrucción, la mayoría de varones y mujeres se encuentran en el nivel medio de agresividad, pero también se observa que entre los niveles alto y muy alto la muestra sobrepasa el $30 \%$, siendo más notable que los varones de quinto grado alcanzan el 40,7\%. Además sumados los niveles bajo y muy bajo, solo en el caso de mujeres de tercer y quinto grado alcanzan 35,8\% y $37,2 \%$ respectivamente. Estos datos se contradicen con los de Matalinares y col (2010), Matalinares y col (2012) quienes encontraron que los varones tienden a ser más agresivos fisica y verbalmente, aunque las mujeres presentan más ira y hostilidad. Resultados análogos, han sido reportados por Castrillon y Vieco (2002) en Medellín. Estos investigadores hallaron también que los hombres tienden a manifestar más agresión física y directa, mientras que las mujeres optan por una agresión verbal e indirecta.

Ahora bien, todos estos hallazgos constituyen un valioso material para su análisis. Veamos ¿La mayor agresividad de los varones, reportada en los estudios aludidos, es una característica genética propia de los varones cómo propuso Lorenz (1966)? Esta tesis, tendría un asidero histórico ya que en la filogénesis de la especie, los machos tendrían que haber desarrollado mayor agresividad que las hembras para defender a la manada. Se han hecho observaciones interesantes al respecto en gorila (Fossey 1983). A este respecto, un estudio de Romaní, Gutiérrez y Lamas (2011) en el Perú, encontró que el perfil del escolar agresor, entre otras cosas, supone el ser varón de 14 a 16 años, precisamente los grupos etarios con los que aquí se trabajó. Pero, la agresividad registrada podría ser también una característica general de la sociedad que la cultura refuerza en los miembros varones de la población. Es particularmente preocupante que nuestros hallazgos se diferencien de los reportes aludidos porque al parecer, las mujeres presentan ahora niveles equivalentes de agresividad al de los varones aunque es claro que los baremos de la prueba no son iguales para unos y otros. Es posible también que por lo menos en parte las diferencias anotadas entre nuestros hallazgos y los de los investigadores mencionados tengan que ver con este aspecto. La teoría de aprendizaje observacional de Bandura, hace hincapié en cómo las condiciones sociales y ambientales enseñan a los individuos a ser agresivos. De hecho los estudios de Noroño y cols. (2002), han puesto de manifiesto la importancia de la familia en la agresividad de los hijos. Así familias disfuncionales y desintegradas tienden a producir hijos más agresivos. Muñoz, (2002) ha reportado un estudio en el que se encontró que los adolescentes agresivos presentan déficits cognitivos en el procesamiento de la información social, tanto en situaciones hipotéticas, como en situaciones reales vividas por los propios adolescentes, en el razonamiento moral presentan un estadío menos desarrollado, así como manifiestan una menor valoración de la solidaridad y baja autoestima. A este respecto, es notable que en la sociedad cada vez más las mujeres se incorporan a las pandillas callejeras, participan en bandas para cometer asaltos 
e incluso asesinatos de modo tal que, la agresividad y violencia no son patrimonio exclusivo de los varones.

Por otro lado, hay que recordar que la teoría de Dollard y Miller (1950) (citado por Soria y Hernández, 1994) propone que la frustración genera irá y ésta dispone al individuo a la agresividad. ¿Es posible que la sociedad peruana esté generando altos niveles de frustración que permiten explicar por lo menos, en parte los altos índices de agresividad registrados cuando se hace un corte a la muestra por grados de instrucción? Según los referidos autores, la frustración surge cuando algo 0 alguien se interpone entre lo que necesitamos 0 deseamos. En este sentido conviene recordar que en el Perú, si bien es cierto que los indicadores macro económicos son "halagüeños para la economía nacional", no lo son así para los sectores pobres que en las últimas décadas se han vuelto más pobres aun y la brecha entre el que tiene y no tiene a crecido de modo ostensible. Por lo demás de acuerdo con Bandura (1973) (citado por Soria y Hernández, 1994) ciertos comportamientos agresivos están recompensados socialmente. Así pues la acción de los factores socioculturales, no deben ser soslayados en este análisis. Sobre todo porque nos guste o no, el Perú, es un país que aunque es pluricultural y multilingüe, tiene como rasgo unificador un marcado machismo.

Por todo ello, en la actualidad resulta difícil sostener la idea de que un fenómeno como la agresividad puede explicarse por un solo factor. De modo que, es más plausible suponer que diversas variables interactúan para dar el resultado que aquí comentamos. Precisamente esto permitiría entender porqué cuando se analizan los niveles de agresividad ya no por grado de instrucción, sino más bien por regiones naturales, el panorama es similar. En efecto, vistos los resultados desde la perspectiva señalada encontramos que en la costa, varones y mujeres se agrupan, casi en todos los casos en el nivel medio de agresividad, excepto en Piura, en que predomina el nivel alto en varones y en Chiclayo, donde predomina el nivel bajo en mujeres. A la vez, los varones de Lima, Trujillo y Piura alcanzan elevados niveles de agresividad, alcanzando hasta el $50 \%$ de la muestra. Estos resultados coinciden con el incremento de la violencia y delincuencia en las ciudades señaladas.
Algo parecido ocurre en la sierra, en lo concerniente al predominio del nivel medio en ambos géneros, resaltando una importante frecuencia entre el nivel alto y muy alto de agresividad de los varones de las ciudades de Ayacucho, Huancayo y Cuzco. Asimismo, las mujeres alcanzan un poco más del $20 \%$ siendo las huancaínas las más agresivas con 39\%. En la selva se observa también el predominio del nivel medio en varones y mujeres aunque en la ciudad de Pucallpa, los varones con altos niveles de agresividad igualan a los de nivel medio. Estas diferencias en función de las ciudades, reforzarían la idea de la influencia de los factores socioculturales. No obstante abre la interrogante acerca de ¿qué factores en esas ciudades están marcando las diferencias apuntadas?

En síntesis, nuestros resultados ratificarían la idea de que la agresividad, se relaciona de diferente modo con las diversas variables sociales y biológicas, por consiguiente exige mirar la situación desde diversos ángulos.

Detengámonos ahora, en el análisis de la variable adicción a la internet. Lo primero que salta a la vista es que en varones y mujeres, cuando se analiza el fenómeno por grado de instrucción predomina el nivel medio con porcentajes muy cercanos al 50\%, lo cual parece indicar que la tendencia a la adicción a la internet no distingue sexo ni grado de instrucción. En este punto, conviene recordar que García y López (2009) han señalado que existe una diversidad de factores de riesgo en las características personales y psicosociales de los usuarios que abusan del uso de internet que incluyen ciertos trastornos psicológicos o adicciones en familiares. Nuestros hallazgos, se contradicen parcialmente con los de Tejeiro (1998) quién encontró mayor tendencia a la adicción en los varones que en las mujeres. Pero, lo que es realmente preocupante, es que la muestra que estudiamos, en ambos grupos, más del $20 \%$ en cada grado, se ubica entre alto y muy altos niveles de adicción lo que significaría que más de la quinta parte de la muestra es adicta. Al respecto, Menéndez (2005) señaló que, los jóvenes susceptibles a tener problemas de adicción a la internet son los introvertidos, con baja autoestima, con poca comunicación familiar, o problemas familiares. Además se ha observado que los adictos a la red (Gil y Vida, 2007) tienden a descuidar la vida familiar, dejar de lado objetivos personales, sentirse mal cuando 
no se realiza la actividad preferida, despreocuparse de sí mismo, adaptarse a un estilo de vida solitario, insensibilizarse ante los problemas de los otros, e incluso tener problemas físicos.

Por otro lado, es notable que nuestros resultados superan los valores reportados por Tejeiro y también largamente los hallados por un equipo de investigadores (2011) de la Universidad de Yale, quienes encontraron aproximadamente un 4\% de adicción a la internet.

Pero el panorama se hace más preocupante aun, cuando lo observamos desde la perspectiva de las regiones naturales. Como era de esperar en todas las ciudades de la costa la mayor frecuencia y porcentaje se ubica en el nivel medio. No obstante, si observamos con mayor acuciosidad, notaremos que en Chiclayo más del 20\% de los varones se encuentra por lo menos en el nivel alto y se muestra una tendencia creciente en ciudades como Piura (24\%), Lima que pasa el 29\%, y casi iguala a Tacna que tiene poco más del $30 \%$, pero lo que es verdaderamente alarmante, es que en la ciudad de Trujillo, los estudiantes que se ubican entre alto y muy alto nivel de adicción a la internet, alcanzan poco más del $45 \%$. Hay que recordar que en esta ciudad también el índice de agresividad es elevado, especialmente entre los varones. En las mujeres, el predominio del nivel medio se mantiene y aunque los niveles alto y muy alto no alcanzan las dimensiones que en los varones, en casi todas las ciudades, las estudiantes se hallan entre el 20 y 30\% con excepción de Lima, que alcanza poco más del $13 \%$. Esto significaría que en la costa si bien las mujeres no alcanzan la proporción de adicción (en sus niveles alto y muy alto) que los varones, presentan porcentajes que no pueden ni deben ser soslayados.

Algo parecido, aunque con sus peculiaridades, se observa al examinar los resultados de la sierra. Ayacucho y Cuzco, tienen más del $50 \%$ de sus jóvenes en el nivel medio de adicción a la internet, lo cual no es algo de lo que debamos dejar de preocuparnos. Es particularmente notable que las ciudades de Huancayo y Huancavelica, tengan más del 30\% de la muestra, ubicados en los niveles alto y muy alto. Esta misma tendencia se observa en el caso de las mujeres, es decir casi la tercera parte de la muestra, por lo menos tiene altos niveles de adicción a la red.

En la selva al examinar los varones, la tendencia al predominio de los niveles medio se mantiene al igual que en las mujeres, pero aquí, los niveles alto y muy alto no alcanzan la proporción que en las ciudades de la costa y la sierra, siendo el departamento de San Martín, el que acumula el más alto porcentaje en varones; aunque por supuesto las frecuencias y porcentajes registrados en los demás, no son despreciables, pues van del 10 al 20\%. En el caso de las mujeres, la ciudad de Pucallpa destaca por que más de la tercera parte de las estudiantes se encuentra en los niveles alto y muy alto. Por todo lo cual, al parecer, varones y mujeres de la selva tendrían un diagnóstico ligeramente más favorable que el correspondiente a los estudiantes de la costa y sierra.

En este sentido, en el ya referido trabajo de Mantalinares y col se sugiere que los factores de riesgo para desarrollar una adicción al internet serían el género masculino, vivir en la costa del Perú, por el fácil acceso al internet sin supervisión, tener entre 15 y 16 años y presentar una alta agresividad. Los autores señalan que estos factores pueden ayudar a adquirir, desarrollar y mantener una adicción a la internet.

Finalmente, es momento de detenernos en las hipótesis de trabajo que fueron sometidas a prueba, se ha supuesto que la adicción a la intenet sería uno de los factores que influye en la agresividad de los estudiantes, varones y mujeres, de tercero a quinto grado de secundaria de las tres regiones naturales: costa, sierra y selva del Perú. Ahora bien, si nuestras suposiciones eran correctas siguiendo la lógica del diseño causal comparativo (Sanchez y Reyes, 2002, Yarlequé, Moya y col, 2007, Yarlequé y Cerron, 2011, Moya, Yarlequé y Cencia, 2012 ) los estudiantes con altos niveles de adicción, debían diferenciarse en su agresividad de aquellos que presentaban bajos niveles de adicción.

Es así, como se formularon las correspondientes hipotesis nulas y alternas. Los resultados, mostraron que en casi todos los casos habían diferencias significativas, entre las medias de agresividad de los sujetos con altos niveles de adicción y los que presentaban bajos niveles de ésta. La consistencia de tales resultados permiten pues, suponer con legitimidad que efectivamente la adicción a la red tendría un papel muy importante en los niveles de agresividad que acusan los jóvenes de la muestra.

Nuestros hallazgos se relacionan con los de Yen, Ko, Yen, Chen, Chung y Chen (2008) quienes encontraron 
entre otras cosas relación entre la adicción a la internet y la hostilidad, y dan un paso adelante respecto de los hallazgos de Matalinares y col (2013) quienes encontraron relación positiva entre agresividad y adicción a la internet; ya que permiten una mejor aproximación al establecimiento de posibles relaciones causales entre las variable estudiadas. En este sentido, los resultados expuestos ratificarían la propuesta de García y Sánchez (2005) quienes han señalado que la adicción a la internet conlleva, entre otras cosas, el cambio de humor y la irritabilidad.

¿Pero cómo pueden explicarse los resultados hasta aquí expuestos? Hemos asumido, la multicausalidad de los fenómenos, por consiguiente es menester hacer el intento de una explicación de este tipo.

En primera instancia debemos subrayar que aquí no estamos afirmando que la adicción a la internet determine la agresividad de los estudiantes de secundaria del Perú, lo que sí parece claro es que el hecho de que Matalinares y Col (en preparación) hayan encontrado correlación directa entre ambas variables y que nosotros hayamos registrado que los adictos a la internet tienen significativamente más elevados niveles de agresividad que los no adictos, nos da razones plausibles para suponer que ésta (la adicción a la internet) tendría un nivel de influencia sobre aquella (la agresividad).

No obstante, la misma adicción, supone ciertas características de personalidad del individuo y ciertas condiciones familiares y sociales en general, a las que hemos hecho referencia líneas arriba. Pero en el problema que nos ocupa, se suma el hecho de que en la educación peruana cada vez más hay menos actividades alternativas para los adolescentes. En efecto el niño y el adolescente de hoy desarrollan cada vez menos actividad física y sus juegos se reducen al accionar de sus dedos frente a la pantalla del ordenador y para incrementar esta situación, desde hace varios años, las gestiones de los ministros de educación, vienen postergando la educación física en las instituciones educativas; con lo cual se agrava el problema de la falta de actividades alternativas. En tales condiciones ¿Cómo no va a incrementarse la adicción a la internet en el Perú?

Por otro lado, los adolescentes con hogares disfuncionales que ingresan profusamente a la internet, ¿a qué páginas y programas acceden? En realidad carecemos de información detallada al respecto, pero es de suponer que en la mayoría de los casos no es para buscar información cultural o científica, sino más bien a los juegos, muchos de ellos violentos y páginas preferentemente de contenido pornográfico (Posada, 2005).

Ahora bien no puede perderse de vista que la sociedad peruana se torna cada vez más violenta, y los jóvenes son cada vez más los principales actores. En este sentido, no es posible soslayar el hecho de que en el Perú a la violencia política y militar de los 80 le sucedió la violencia delincuencial y que al parecer nos encaminamos a una etapa de coexistencia de ambas formas de violencia ante la inoperancia e ineficiencia del Estado. En consecuencia queda claro que los jóvenes de hoy son herederos de una sociedad y cultura violenta de modo pues que sería erróneo querer achacar toda la responsabilidad de la agresividad registrada en los jóvenes a la internet.

Finalmente no puede descuidarse en este análisis el enfoque del aprendizaje. Veamos: Hace tres décadas en el Perú, las pandillas callejeras, eran cosas que veíamos en la televisión y en el cine. Los asesinatos Ilevados a cabo por menores de edad en las calles e incluso en las instituciones educativas eran, hasta hace unos años, cosas de las que nos enterábamos por las noticias pero hoy son una realidad en el Perú y al parecer una buena parte de esto a sido aprendido por modelos, más aun cuando el sistema social, político y económico del país, refuerza con la impunidad tales acciones y genera constantemente las condiciones para que cada vez más jóvenes se hagan delincuentes.

Así pues en nuestra opinión la adicción a la internet no es más que una expresión de los riesgos que puede traer el avance tecnológico para los jóvenes sin control. La agresividad es una de sus consecuencias. Pero no es la única y la agresividad misma tiene en su base muchos otros factores que la acicatean.

\section{REFERENCIAS BIBLIOGRÁFICAS}

- Arai, C.; González, C. Morales, D. Gutiérrez, F. yTettley, C. Consecuencias del uso de Internet en alumnos de preparatoria. Recuperado el 28 de octubre del 2011 en:http://www.acmor.org.mx/cuam

- Balaguer, R. 2001, "La adicción a Internet”. Fuente original: Adicción, Montevideo: Cuadernos de la Coordinadora de Psicólogos del Uruguay. Disponible en el ARCHIVO del Observatorio para la Ciber 
Sociedad en http://www.cibersociedad.net/archivo/ articulo.php?art=2

- Berkowitz (1996). Agresión. Causas, consecuencias y control. Bilbao: Descleé de Briuwer.

- Ornella V., Matalinares M. y Deyvi. R (2012) Propiedades psicométricas del test de adicción al internet. Revista de investigaciones psicológicas. Vol 15.

- Matalinares M. y Gloria Díaz A. Carlos Arenas I., Alejandro Dioses Ch., Nicolas Medina C., Ornella Raymundo V., Deyvi Baca R., Erika Fernández A., Joel Uceda E., Yasmin Huari T., Nayda Villavicencio C., Persin Vargas V., Milagros Quispe N., Elsa Sánchez Y., Emanuel Leyva O. y Ailin Díaz V. (2012)ADICCION AL INTERNET Y AGRESIVIDAD EN ESTUDIANTES DE SECUNDARIA DEL PERU. Instituto de investigación de la faculta de psicología de la Universidad Mayor de San Marcos.
- Rodríguez, J., Peña, E. y Graña, J. (2002). Adaptación psicométrica de la versión española del Cuestionario de Agresión. Psicothema, 14 (2), 476-482.

- Yen, J., Ko, C., Yen, C., Chen, S., Chung, W., y Chen, C. (2008). Psychiatric symptoms in adolescents with Internet addiction: Comparison with substance use. Psychiatry and Clinical Neurosciences, 62, 9-16.

- Young, K. S. (1996). Internet addiction:The emergence of a new clinical disorder. Artículopresentado en la 104th annual meeting of the American Psychological Association, August 11, 1996. Toronto, Canada.

- Young, K.S. y Rodgers, R.C. (1998). Internet addiction: Personality traits associated with its development. Artículopresentado en el 69th annual meeting of the Eastern Psychological Association.

- Trujillo,H.,Gonzales,J.,León,C.,Valenzuela,C. y Moyano,M.(2006).De la agresividad a la violencia 